\title{
The effect of Sevoflurane vs. Isoflurane on Emergence Time and PACU Length of Stay
}

\author{
Kamal Maheshwari, Sanchit Ahuja, Edward J. Mascha, Praveen Chahar, Kenneth Cummings, \\ Hesham Elsharkawy, Daniel I. Sessler, Andrea Kurz \\ Outcomes ReseARCH Department, Anesthesiology Institute, Cleveland Clinic, Cleveland, United States
}

\section{Background}

In ideal operating room setting, the goal is to minimize emergence timing and post anaesthesia care unit (PACU) length of stay, thus reducing cost. ${ }^{1}$ With the routine use of shorter acting inhalational anaesthetic agent quicker emergence and shorter PACU length of stay seems to be possible. We therefore assessed the treatment difference of Isoflurane versus Sevoflurane on PACU length of stay and emergence timing.

\section{Methods}

We included 1498 adults from a previously conducted prospective alternating intervention trial ${ }^{2}$, mostly having colorectal surgery, who were assigned to isoflurane or sevoflurane based on protocol. The PACU length of stay was defined as the time from entering to exiting PACU phase I. Emergence time was defined as the time from last 0.3 (or higher) MAC inhalational dose towards the end of the procedure to out of the operating room. Treatment effect was assessed using Cox proportional hazards regression and adjusting for imbalanced baseline variables. In a post-hoc analysis we compared the two anaesthetics by type of surgery.

\section{Results}

PACU length of stay did not differ between groups, with a median [quartiles] of $158[117,216]$ minutes for isoflurane and $155[118,219]$ minutes for sevoflurane group; the adjusted hazard ratio for PACU discharge time was $1.03(95 \% \mathrm{CI} ; 0.92,1.16)(\mathrm{P}=0.53)$. However, isoflurane did have shorter PACU length of stay for non-abdominal surgeries. Emergence timing was longer for isoflurane, with median [quartiles] of 16 [12, 22] minutes versus 14 [11, 19] minutes for sevoflurane, with adjusted hazard ratio of 0.80 (95\% CI: $0.72,0.90)$, $\mathrm{P}<0.001$. Figure-1, Table 1.

\section{Conclusion}

Use of inhalational agent isoflurane versus sevoflurane had no effect on PACU length of stay. Emergence timing is on average about two minutes quicker with sevoflurane, which may be of limited clinical significance. Sevoflurane, per Minimum Alveolar Concentration (MAC) hour, is twelve times more expensive compared to isoflurane and thus offer no advantage with respect to PACU length of stay and emergence timing.

\section{Figure 1}

Hazard Ratio (97.5\% CI) (Iso vs Sevo)

Emergence Timing (min)

PACU Length of Stay (min)

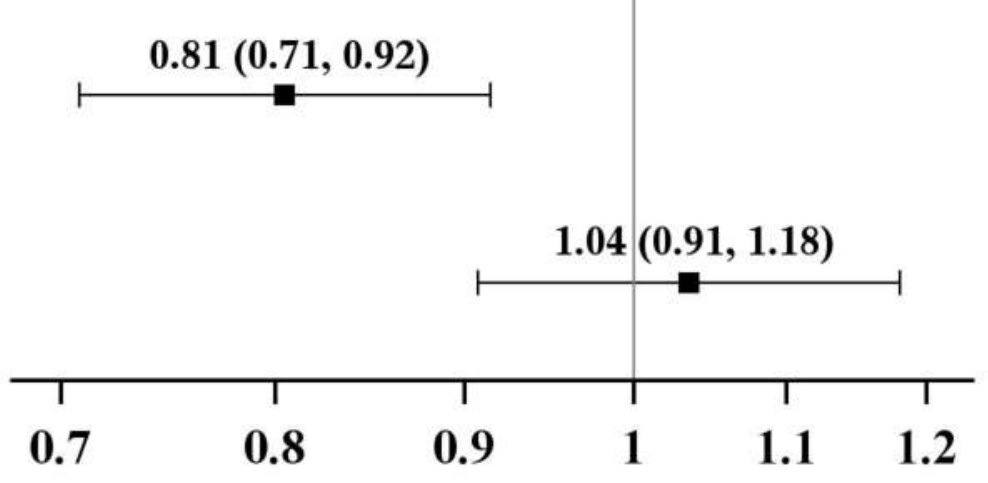

\begin{tabular}{|c|c|c|c|c|}
\hline & & & & \\
\hline Factor & $\begin{array}{c}\text { Iso } \\
(\mathrm{N}=674)\end{array}$ & $\begin{array}{c}\text { Sevo } \\
(\mathrm{N}=\mathbf{8 2 4})\end{array}$ & $\begin{array}{c}\text { Hazard Ratio } \\
\text { (97.5\% CI) } \\
\text { (Iso vs Sevo) }\end{array}$ & p-value \\
\hline \multicolumn{5}{|l|}{ Primary Analysis } \\
\hline Emergence Timing (min) & $16[12,22]$ & $14[11,19]$ & $\begin{array}{l}0.81(0.71 \\
0.92)\end{array}$ & 0.0001 \\
\hline $\begin{array}{l}\text { PACU Length of Stay } \\
\text { (min) }\end{array}$ & $158[117,216]$ & $155[118,219]$ & $\begin{array}{c}1.04(0.91 \\
1.18)\end{array}$ & 0.56 \\
\hline \multicolumn{5}{|l|}{ Secondary Analysis } \\
\hline \multicolumn{5}{|l|}{$\begin{array}{l}\text { PACU Length of Stay } \\
(\mathrm{min})\end{array}$} \\
\hline \multirow[t]{2}{*}{ Abdominal Surgery } & $(\mathrm{N}=466)$ & $(\mathrm{N}=564)$ & & \\
\hline & $173[131,229]$ & $167[128,231]$ & $\begin{array}{c}0.95(0.82 \\
1.11)\end{array}$ & 0.48 \\
\hline \multirow[t]{2}{*}{ Non-Abdominal Surgery } & $(\mathrm{N}=\mathbf{2 0 8})$ & $(\mathrm{N}=\mathbf{2 6 0})$ & & \\
\hline & $123[95,166]$ & $134[98,177]$ & $\begin{array}{c}1.32(1.05 \\
1.66)\end{array}$ & 0.006 \\
\hline
\end{tabular}

* Hazard ratio was estimated from cox proportional hazards model. Higher hazard indicates earlier discharge from PACU or earlier emergence.

$\wedge$ For primary analysis, the model was adjusted for imbalanced variables (ASD $>0.10$ ), including ASA Physical Status, Weight Loss, Procedure, Anesthesia Provider, Surgeon and Time-weighted Average End-tidal Volatile. Significance level was 0.025

\# For secondary analysis, the model included the interaction term between treatment and abdominal surgery, adjusting for the same imbalanced variables except for procedure type. Significance leve was 0.025 . The interaction between abdominal surgery and treatment was significant for PACU length of stay $(P=0.01)$, while not significant for emergence time $(P=0.86)$

Statistics presented as Mean \pm SD, Median [P25, P75], Median (min, max) or N (column \%). p-values: $a=A N O V A, b=$ Kruskal-Wallis test, $c=$ Pearson's chi-square test, $d=$ =Fisher's Exact test. $\wedge$ as Morphine IV Equivalent

\# Showed Top 10 frequency ones.

* The pain score is a $0-10$ verbal response scale

\section{References}

1. Smith I: Cost considerations in the use of anaesthetic drugs. Pharmacoeconomics 2001; 19: 469-8

2. Kopyeva T, Sessler DI, Weiss S, Dalton JE, Mascha EJ, Lee JH, Kiran RP, Udeh B, Kurz A: Effects of volatile anesthetic choice on hospital length-of-stay: a retrospective study and a prospective trial. Anesthesiology 2013; 119: 61-70 